\title{
Surveillance de la maladie de Lyme au Canada, de 2009 à 2012
}

\author{
Ogden N.H..$^{*}$, Koffi $J^{1}$, Lindsay $L^{2}{ }^{2}$, Fleming $S^{3}$, Mombourquette $\mathrm{DC}^{3}$, Sanford $\mathrm{C}^{4}$, Badcock $\mathrm{J}^{5}$, \\ Gad RR ${ }^{5}$, Jain-Sheehan $N^{6}$, Moore $S^{6}$, Russell $C^{6}$, Hobbs $L^{6}$, Baydack $R^{7}$, Graham-Derham $S^{7}$, \\ Lachance $\mathrm{L}^{8}$, Simmonds $\mathrm{K}^{8}$, Scott $\mathrm{AN}^{8}$
}

\begin{abstract}
${ }^{1}$ Centre des maladies infectieuses d'origine alimentaire, environnementale et zoonotique, Agence de la santé publique du Canada, Ottawa (Ontario)

${ }^{2}$ Laboratoire national de microbiologie, Agence de la santé publique du Canada, Winnipeg (Manitoba)

${ }^{3}$ Direction générale de la santé publique, ministère de la Santé et du Mieux-être de la Nouvelle-Écosse, Halifax

(Nouvelle-Écosse)

${ }^{4}$ Surveillance et Évaluation de la santé de la population, ministère de la Santé et du Mieux-être de l'Île-du-Prince-Édouard, Charlottetown, (Île-du-Prince-Édouard)

${ }^{5}$ Bureau du médecin hygiéniste en chef, ministère de la Santé du Nouveau-Brunswick, (Nouveau-Brunswick)

${ }^{6}$ Maladies entériques, zoonotiques et à transmission vectorielle, Santé publique Ontario, Toronto (Ontario)

${ }^{7}$ Direction générale de la lutte contre les maladies transmissibles, Vie saine et Aînés, Santé Manitoba, Winnipeg (Manitoba)

${ }^{8}$ Direction générale de la surveillance et de l'évaluation, ministère de la Santé de l'Alberta, Calgary (Alberta)
\end{abstract}

*Correspondance : Nicholas.ogden@phac-aspc.gc.ca

\section{Résumé}

Objectifs : Résumer les quatre premières années de la surveillance à l'échelle nationale de la maladie de Lyme au Canada de 2009 à 2012, et effectuer une comparaison préliminaire des manifestations cliniques de la maladie au Canada et aux États-Unis

Méthodologie : Calcul du nombre et du taux d'incidence des cas déclarés, par province, mois, année, âge et par sexe. Un modèle de régression logistique a été utilisé pour examiner les tendances au fil du temps. Les lieux d'acquisition ont été cartographiés et les manifestations cliniques ont été signalées pour les compétences pour lesquelles des données étaient disponibles. Des écarts entre les provinces, par année, par âge et par sexe, ainsi que la présence des symptômes cliniques ont été examinés en régression logistique. Une première analyse comparative s'est penchée sur les symptômes présentés au Canada et aux États-Unis.

Résultats : Le nombre de cas signalés a augmenté considérablement, soit de 144 en 2009 à 338 en 2012 (coefficient de corrélation $=0,34$, erreur standard $=0,07, P<0,05$ ), ce qui est principalement attribuable à une augmentation de l'incidence des infections contractées au Canada. Plus de cas ont été classés dans la catégorie " confirmé » $(71,5 \%)$ que dans la catégorie " probable » (28,5\%). La plupart des cas sont survenus dans des zones où la présence de populations de tiques vectrices avait été établie. Plus d'hommes que de femmes ont été infectés (53,4 \% par rapport à 46,6\%), et l'incidence était la plus élevée chez les adultes âgés de 55 à 74 ans et chez les enfants âgés de 5 à 14 ans. La plupart des cas (95\%) ont été acquis d'avril à novembre. Des cas d'infection contractée dans les régions endémiques, 39,7\% présentaient des signes de la maladie de Lyme au stade précoce, alors que 60,3 \% manifestaient des symptômes de la maladie de Lyme au stade disséminé. Des différences importantes ont été observées entre les groupes d'âge, les sexes et les provinces en ce qui a trait à la fréquence du signalement de symptômes cliniques. La proportion de cas d'infection contractée dans les régions endémiques et de la maladie de Lyme au stade précoce était inférieure à la proportion signalée aux États-Unis.

Conclusion : L'incidence de la maladie de Lyme est à la hausse au Canada. La plupart des cas sont contractés dans les régions où les populations de tiques vectrices sont en croissance et cela varie géographiquement dans une même province et d'une province à l'autre. Il y a aussi des différences de fréquence selon l'âge, la saison et les symptômes. La plus faible proportion de cas au stade précoce de la maladie de Lyme au Canada comparativement aux États-Unis semble indiquer une plus faible sensibilisation à l'égard de la maladie de Lyme au stade précoce au Canada, mais cela exige une étude plus approfondie. 


\section{Introduction}

La maladie de Lyme, causée par la bactérie Borrelia burgdorferi sensu stricto en Amérique du Nord, est transmise aux humains par des animaux sauvages réservoirs hôtes de tiques Ixodes spp. (1) dans leur habitat boisé (2). Le risque de contracter la maladie de Lyme au Canada survient dans les endroits du sud de la Colombie-Britannique où les tiques vectrices sont établies (où est établie la tique Ixodes pacificus, vecteur relativement inefficace), et dans les régions méridionales du centre et de l'est du Canada où se propage la tique Ixodes scapularis, un vecteur efficace provenant des États-Unis et provoquant l'apparition de la maladie de Lyme au Canada (3). Le risque de contracter la maladie de Lyme touchant une plus grande zone géographique est faible, puisque les tiques sont transportées par les populations d'oiseaux migrateurs $(4,5)$.

À la lumière de la documentation concernant la migration vers le nord de tiques au Canada, la maladie de Lyme est devenue une maladie à déclaration obligatoire au Canada en 2009 et des renseignements de base sur les cas humains sont soumis par l'ensemble des provinces et des territoires au Système canadien de surveillance des maladies à déclaration obligatoire (SSMDO) coordonné par l'Agence de la santé publique du Canada (ASPC). Une initiative de surveillance accrue de la maladie de Lyme a été lancée par l'Agence de la santé publique en 2010 en collaboration avec des organismes provinciaux de santé publique afin d'obtenir davantage de données détaillées sur les cas de maladie de Lyme. Ensemble, ces systèmes de surveillance visent à déterminer les tendances changeantes en matière d'incidence de la maladie de Lyme, la population canadienne à risque et les types de maladie clinique au Canada en vue d'éclairer le diagnostic et le signalement de la maladie de Lyme par les médecins cliniciens.

Dans le cadre de cette étude, les données recueillies au cours des quatre premières années de la surveillance à l'échelle nationale de la maladie de Lyme (de 2009 à 2012) sont présentées et analysées pour décrire les profils précoces d'apparition de la maladie de Lyme au Canada. Puisque l'émergence de la maladie de Lyme dans le centre et l'est du Canada constitue probablement un élargissement de l'émergence de la maladie de Lyme aux États-Unis, les profils des cas de la maladie de Lyme (âge, saison de l'acquisition et manifestations) ont été comparés à ceux qui ont été signalés aux États-Unis.

\section{Méthodologie}

\section{Sources des données sur les cas d'infection humaine}

Les données du SSMDO sur le nombre annuel de cas signalés à tous les organismes de santé publique provinciaux et territoriaux par les médecins cliniciens ou par les laboratoires provinciaux étaient disponibles pour la période de 2009 à 2012. Les renseignements de base signalés comprenaient le sexe, l'âge et la date de l'épisode. Ces cas étaient classés dans la catégorie « confirmé » ou " probable » par les organismes de santé publique provinciaux soumettant les données, à l'exception de la Colombie-Britannique, du Québec et du Nouveau-Brunswick qui ont déclaré tous les cas sans classification et n'ont pas signalé de cas d'érythème migrant sans confirmation en laboratoire du diagnostic.

\section{Définition des cas aux fins de surveillance de la maladie de Lyme à l'échelle nationale (6)}

\section{Cas confirmé}

Manifestation clinique de la maladie et confirmation en laboratoire par :

- l'isolement de Borrelia burgdorferi dans un échantillon clinique approprié;

- la détection d'ADN de $B$. burgdorferi par réaction en chaîne de la polymérase;

- un test sérologique positif selon les critères de l'approche en deux étapes (épreuve ELISA et méthode de transfert Western blot) avec antécédents de résidence ou de voyage dans une région d'endémicité. 


\section{Cas probable}

P1 = un test sérologique positif selon les critères de l'approche en deux étapes (épreuve ELISA et méthode de transfert Western blot) sans antécédents de résidence ou de voyage dans une région d'endémicité*

OU

P2 = érythème migrant observé par un clinicien en l'absence de résultats de laboratoire indiquant une infection, mais avec antécédents de résidence ou de voyage dans une région d'endémicité de la maladie de Lyme. ${ }^{1}$

\footnotetext{
${ }^{1}$ Par région d'endémicité, on entend une zone où la présence d'une population de tiques a été démontrée (par plusieurs visites sur place) et où il a été établi que ces tiques favorisaient la transmission de B. burgdorferi chez les animaux sauvages hôtes (7). De plus, en raison du coût de plusieurs visites sur place, le risque environnemental pour la maladie de Lyme est défini comme étant des zones « à risque » où la présence de tiques a été détectée par la surveillance sur le terrain, mais non confirmée par plusieurs visites sur place (3).
}

En 2010, le système de surveillance accrue de la maladie de Lyme a été mis sur pied en partenariat avec les provinces du Manitoba, de l'Ontario, du Nouveau-Brunswick et de la Nouvelle-Écosse. En 2012, l'Alberta, la Saskatchewan et l'Île-du-Prince-Édouard se sont jointes. Les données transmises dans le formulaire standard du système de surveillance accrue de la maladie de Lyme comprenaient des renseignements sur la possibilité d'acquisition de l'infection à l'intérieur ou à l'extérieur du Canada, les détails des manifestations cliniques et des méthodes de diagnostic en laboratoire. On a noté des différences entre les provinces participant au système de surveillance accrue de la maladie de Lyme quant aux données fournies (Annexe 1).

\section{Manifestations cliniques}

De l'information sur les caractéristiques cliniques a été fournie par le Manitoba, l'Ontario, le Nouveau-Brunswick et la Nouvelle-Écosse, bien que le Manitoba n'ait signalé que deux catégories de symptômes : érythème migrant et « autres manifestations cliniques », c'est-à-dire des données probantes de la maladie de Lyme au stade disséminé, mais sans plus de renseignements sur les symptômes. Les catégories de manifestations cliniques étaient celles de la maladie de Lyme au stade précoce (c.-à-d. un érythème migrant), au stade disséminé précoce, y compris les manifestations de la neuroborréliose (paralysie de Bell ou autres manifestations neurologiques de la maladie de Lyme au stade disséminé), les manifestations cardiaques et les manifestations de la maladie disséminée tardive telles que l'arthrite. (Voir l'encadré ci-dessous.) II est à noter que l'on a supposé que tous les cas probables « $\mathrm{P} 2$ » ( $\mathrm{p}$. ex. les cas présentant un érythème migrant, mais sans résultat de test sérologique) étaient des cas au stade précoce de la maladie de Lyme présentant une seule lésion d'érythème migrant plutôt que plusieurs lésions (qui surviennent au stade disséminé de la maladie de Lyme). On pourrait s'attendre à ce que les cas présentant plusieurs érythèmes migrants présentent des résultats de tests sérologiques positifs et soient classés comme des cas confirmés ou des cas probables « P1 ».

\section{Manifestations cliniques de la maladie de Lyme (8)}

Stade localisé précoce : érythème migrant + / - fièvre, arthralgie et céphalées

Stade disséminé : érythème migrant multiple + / - fièvre, arthralgie, céphalées et lymphadénopathie

Troubles cardiaques : antiviraux, tachyarythmies, myopéricardite, dysfonction myocardique

Troubles neurologiques : méningite à liquide clair, neuropathie crânienne (p. ex., paralysie de Bell), radiculopathie avec atteinte motrice ou sensorielle

Stade disséminé tardif : arthrite oligoarticulaire

Troubles neurologiques : encéphalopathie, polyradiculoneuropathie axonale, encéphalomyélite chronique ${ }^{1}$

${ }^{1}$ Les manifestations neurologiques du stade tardif de la maladie de Lyme sont très rares (9), et il en est de même en ce qui concerne la collecte et le transfert de données; ainsi, tous les cas de la maladie de Lyme présentant des manifestations neurologiques ont été considérés comme étant du stade disséminé précoce de la maladie de Lyme. 


\section{Analyse des données}

Les données du Système de surveillance accrue de la maladie de Lyme et du SSMDO ont été résumées et, dans la mesure du possible, comparées aux données similaires provenant des États-Unis (10), où des données similaires ont été recueillies pendant plus de 20 ans. L'incidence annuelle au Canada, ainsi que l'incidence par province, sexe et âge ont été calculées par populations de 100000 habitants. Les dénominateurs étaient des estimations de la population de recensement en date du $1^{\mathrm{er}}$ juillet, pour chaque année, de 2009 à 2012 (11). La proportion de cas signalés par mois et par classification de cas pour chaque année a également été calculée. Les tendances concernant le nombre de cas signalés à l'échelle nationale pour la période de 2009 à 2012 (cas classés comme " probables " et " confirmés » combinés) ont été examinées en procédant à une estimation par les moindres carrés pondérés dans Stata SE 11.0 pour Windows (College Station, Texas), l'année constituant la variable explicative représentant les dernières estimations de la population canadienne (12). L'analyse a été menée à partir des données sur le nombre de cas et des données déclarées alors, mais celles-ci pourraient varier légèrement en raison de la détermination rétrospective des cas.

L'analyse du nombre de cas endémiques par rapport aux cas liés à des voyages, au lieu d'acquisition et aux caractéristiques cliniques a été effectuée pour les cas signalés par le Système de surveillance accrue de la maladie de Lyme du Manitoba, de l'Ontario, du Nouveau-Brunswick et de la Nouvelle-Écosse.

Les lieux probables d'exposition des cas au Canada ont été cartographiés à l'aide de la version 10.2 du système d'information géographique ArcGIS (ESRI), la localisation des points constituant le centre des régions de tri d'acheminement (Tableau 1) ou les régions où la maladie est endémique, selon le lieu d'acquisition signalé. Les zones endémiques connues et les régions à risque (3) ont également été cartographiées pour effectuer une comparaison visuelle. 
Tableau 1 : Cas de la maladie de Lyme, par classification, et par année, de 2009 à 2012

\begin{tabular}{|c|c|c|c|c|c|c|c|c|c|c|}
\hline \multicolumn{11}{|c|}{ Année } \\
\hline \multirow[t]{2}{*}{ Classification des cas } & \multicolumn{2}{|c|}{2009} & \multicolumn{2}{|c|}{2010} & \multicolumn{2}{|c|}{2011} & \multicolumn{2}{|c|}{2012} & \multicolumn{2}{|c|}{ Total } \\
\hline & $\mathrm{N}$ & $(\%)$ & $\mathrm{N}$ & $(\%)$ & $\mathrm{N}$ & $(\%)$ & $\mathrm{N}$ & $(\%)$ & $\mathrm{N}$ & $(\%)$ \\
\hline \multicolumn{11}{|c|}{ Tous les cas } \\
\hline Confirmés & 115 & $(79,9)$ & 107 & $(74,8)$ & 188 & $(70,7)$ & 227 & $(67,2)$ & 637 & $(71,5)$ \\
\hline Probables & 29 & $(20,1)$ & 36 & $(25,2)$ & 78 & $(29,3)$ & 111 & $(32,8)$ & 254 & $(28,5)$ \\
\hline Total & 144 & 100 & 143 & 100 & 266 & 100 & 338 & 100 & 891 & 100 \\
\hline \multicolumn{11}{|c|}{ Cas d'infection contractée au Canada appuyés par des données cliniques ${ }^{1}$} \\
\hline Confirmés & 44 & $(81,5)$ & 43 & $(68,3)$ & 79 & $(61,7)$ & 93 & $(65,5)$ & 259 & $(66,9)$ \\
\hline Tous les cas probables & 10 & $(18,5)$ & 20 & $(31,7)$ & 49 & $(38,3)$ & 49 & $(34,5)$ & 128 & $(33,1)$ \\
\hline $\begin{array}{l}\text { - Première définition de } \\
\text { cas probable }\end{array}$ & 8 & $(14,8)$ & 15 & $(23,8)$ & 40 & $(31,3)$ & 24 & $(16,9)$ & 87 & $(22,5)$ \\
\hline $\begin{array}{l}\text { - Deuxième définition de } \\
\text { cas probable }\end{array}$ & 2 & $(3,7)$ & 5 & $(7,9)$ & 9 & $(7,0)$ & 25 & $(17,6)$ & 41 & $(10,6)$ \\
\hline Total & 54 & 100 & 63 & 100 & 128 & 100 & 142 & 100 & 387 & 100 \\
\hline
\end{tabular}

${ }^{\mathrm{I}}$ Cas pour lesquels des données cliniques détaillées (symptômes et diagnostic en laboratoire) et les renseignements sur l'exposition étaient disponibles permettant de distinguer les deux définitions différentes de cas probables.

Les écarts entre les provinces et territoires, entre les années, entre les groupes d'âge et entre les sexes, et dans les proportions des différentes manifestations cliniques de la maladie de Lyme au stade disséminé ont été explorés au moyen du modèle de régression logistique dans Stata SE 11.0. Les variables des résultats étaient la présence ou l'absence d'un érythème migrant, de manifestations neurologiques, de manifestations cardiaques et d'œdème articulaire ou d'arthrite dans des modèles distincts. Les variables explicatives de l'âge, de l'année, de la province et du sexe ont été examinées pour la première fois en analyses bivariées, et celles présentant des associations avec le résultat à un niveau de signification de $\mathrm{P}<0,1$ ont été incorporées aux modèles multivariables. Les relations polynomiales entre l'âge et la fréquence des manifestations (en utilisant l'âge et l'âge au carré comme variables explicatives) ont été explorées comme il est suggéré par l'inspection visuelle de graphiques ajustés par la fonction Lowess de ces relations. Les modèles multivariables les plus restreints étaient recherchés par l'élimination de variables non significatives. Le niveau de signification du modèle multivariable était de $P<0,05$. 


\section{Résultats}

\section{Incidence et tendances temporelles}

Le nombre de cas signalés a augmenté considérablement de 144 en 2009 à 338 en 2012 (coefficient de corrélation $=0,34$, erreur standard $=0,07, P<0,05$; tableau 1), et l'incidence a augmenté, passant de 0,4 à 1,0 cas par 100000 habitants (tableau 2).

Tableau 2 : Incidence des cas de la maladie de Lyme, par province, et par année, de 2009 à 2012

\begin{tabular}{|l|c|c|c|c|}
\hline \multirow{2}{*}{ Province } & \multicolumn{5}{c|}{ Année } \\
\cline { 2 - 5 } & $\mathbf{2 0 0 9}$ & $\mathbf{2 0 1 0}$ & $\mathbf{2 0 1 1}$ & $\mathbf{2 0 1 2}$ \\
\hline \multicolumn{7}{|c|}{ Tous les cas } \\
\hline Colombie-Britannique & 0,2 & 0,2 & 0,4 & 0,4 \\
\hline Alberta & 0,0 & 0,0 & 0,2 & 0,2 \\
\hline Saskatchewan & 0,0 & 0,0 & 0,1 & 0,0 \\
\hline Manitoba & 0,4 & 1,0 & 1,0 & 1,5 \\
\hline Ontario & 0,8 & 0,7 & 1,0 & 1,4 \\
\hline Québec & 0,2 & 0,1 & 0,4 & 0,5 \\
\hline Île-du-Prince-Édouard & 0,0 & 0,0 & 0,7 & 1,4 \\
\hline Nouveau-Brunswick & 0,0 & 0,3 & 0,7 & 0,9 \\
\hline Nouvelle-Écosse & 1,7 & 1,8 & 5,7 & 5,4 \\
\hline Terre-Neuve-et-Labrador & 0,0 & 0,2 & 0,0 & 0,0 \\
\hline Canada & 0,4 & 0,4 & 0,8 & 1,0 \\
\hline \multicolumn{2}{|c|}{ Infections contractées au Canada } \\
\hline Saskatchewan & 0,0 & 0,0 & 0,1 & 0,0 \\
\hline Manitoba & 0,3 & 0,6 & 0,6 & 1,0 \\
\hline Ontario & 0,5 & 0,5 & 0,8 & 0,8 \\
\hline Île-du-Prince-Édouard & 0,0 & 0,0 & 0,0 & 0,7 \\
\hline Nouveau-Brunswick & 0,0 & 0,3 & 0,4 & 0,7 \\
\hline Nouvelle-Écosse & 1,5 & 1,5 & 5,2 & 5,3 \\
\hline La Colombie-Britannique et le Québec n'ont pas fourni l'information à savoir si l'infection avait été contractée au Canada ou en voyage à l'extérieur du \\
Canada. Tous les cas en Alberta et à Terre-Neuve-et-Labrador étaient dus à des infections contractées pendant des voyages à l'extérieur du Canada.
\end{tabular}

Des cas ont été signalés dans toutes les provinces, la plupart des infections contractées au Canada survenant en Colombie-Britannique, au Manitoba, en Ontario, au Québec, au Nouveau-Brunswick et en Nouvelle-Écosse. Tous les cas signalés par l'Alberta et Terre-Neuve-et-Labrador ont été déclarés comme étant des infections contractées pendant des voyages à l'extérieur du Canada.

En 2012, l'incidence était inférieure à 1,0 pour 100000 habitants dans cinq provinces, soit au Manitoba, en Ontario, au Nouveau-Brunswick, en Nouvelle-Écosse et à l'île-du-Prince-Édouard (Tableau 2). L'incidence s'est accrue principalement au Manitoba, en Ontario, au Québec, au Nouveau-Brunswick et en Nouvelle-Écosse (Tableau 2). II y a eu une légère diminution du nombre de cas signalés en Colombie-Britannique et en Nouvelle-Écosse, de 2011 à 2012.

La majorité des cas ont été déclarés comme des cas " confirmés " (Tableau 1). Parmi les cas sans antécédents de voyage à l'extérieur du Canada, 387 cas comprenaient des données sur les symptômes cliniques et le lieu probable de l'infection. Pour ces cas (signalés par le Manitoba, l'Ontario, le Nouveau-Brunswick et la Nouvelle-Écosse), le nombre de cas P1 et P2 «probables » pouvait être estimé. Pour ces provinces, la plupart des cas probables étaient de la catégorie P1 (Tableau 1). 


\section{Variation de l'incidence avec l'âge et le sexe}

Les taux d'incidence variaient selon les groupes d'âge, les plus élevés se retrouvant chez les personnes âgées de 55 à 74 ans et chez les enfants (âgés de moins de 18 ans), l'incidence la plus élevée étant signalée chez les 5 à 14 ans (Figure 1). Plus de cas ont été signalés chez les hommes (476/891, 53,4\%) que chez les femmes (409/891, 46,6 \%) dans la plupart des groupes d'âge (16/18) (Figure 1).

Figure 1 : Incidence des cas de la maladie de Lyme signalés pour 100000 habitants de 2009 à 2012, selon l'âge et le sexe

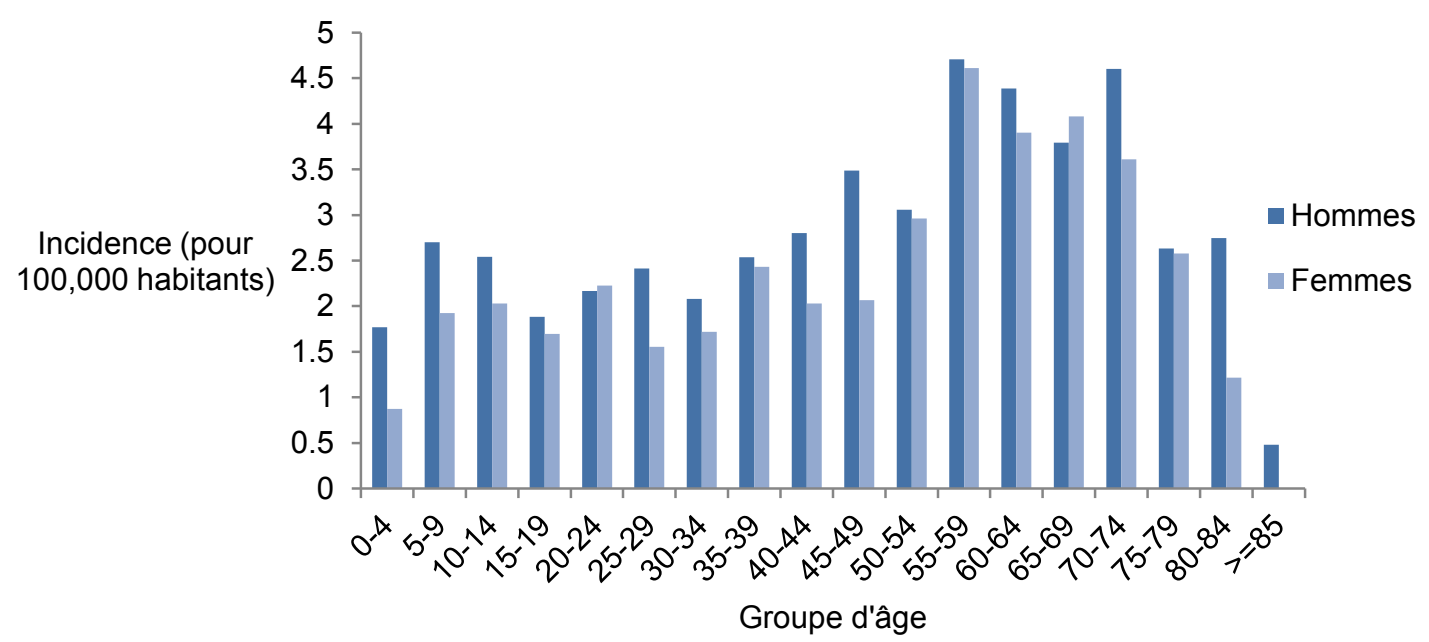

\section{Caractère saisonnier}

Des 387 cas pour lesquels le type d'épisode a été consigné, la date de l'épisode était la date d'apparition de la maladie dans 328 cas $(84,7 \%)$, la date de prélèvement de l'échantillon pour le diagnostic dans 42 cas $(10,8 \%)$, la date de diagnostic clinique ou en laboratoire dans 13 cas $(3,3 \%)$ et la date de déclaration dans 3 cas $(0,8 \%)$. Les cas de la maladie de Lyme étaient associés à des dates d'épisode comprises dans tous les mois de l'année, mais la plupart (544/891, 61,1\%) ont eu lieu de juin à août (Figure 2). Les cas en Colombie-Britannique avaient tendance à se produire plus tôt et plus tard dans l'année que dans d'autres provinces, tandis qu'une plus grande proportion de cas était signalée en octobre au Manitoba comparativement aux autres provinces (Figure 2). 
Figure 2 : Proportion des cas de la maladie de Lyme signalés de 2009 à 2012 et acquis au Canada, par date d'épisode ${ }^{1}$

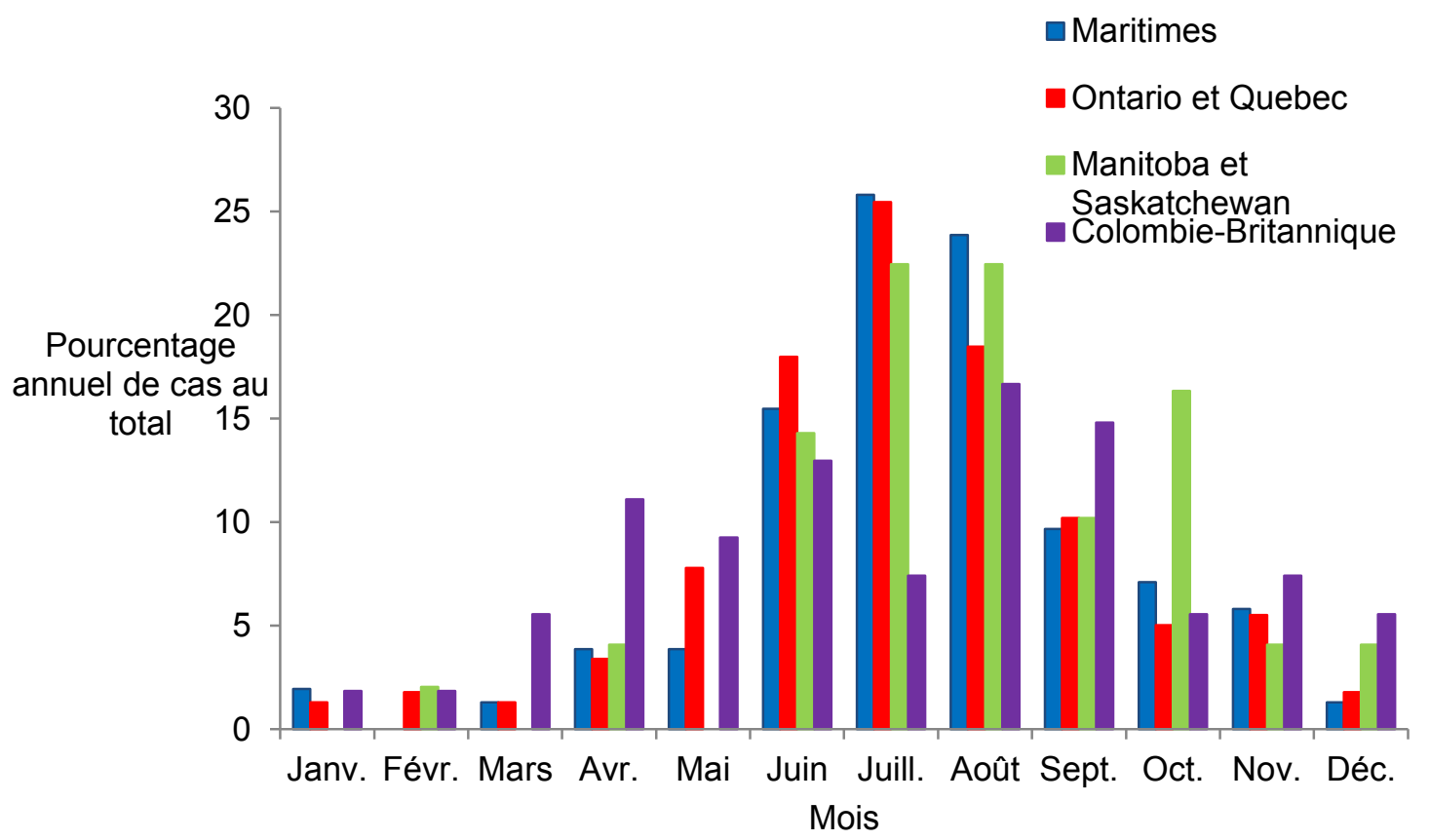

\footnotetext{
${ }^{1}$ Lorsque la date d'apparition de la maladie n'était pas disponible, la date du diagnostic ou de la collecte de l'échantillon pour le diagnostic en laboratoire ont été utilisées.

\section{Emplacement géographique de l'acquisition}

La plupart des infections contractées au Canada ont été contractées dans des zones connues comme des régions endémiques ou à risque, bien que des cas aient été signalés à l'extérieur de ces régions (Figure 3). Le nombre de cas signalés annuellement comme étant été contractés à l'extérieur du Canada entre 2009 et 2012 était stable : de 38 à 48 cas par année de 2009 à 2012. Des 54 cas signalés de 2009 à 2012 pour lesquels le lieu du voyage à l'extérieur du Canada était fourni, 38 (70,4 \%) étaient des infections contractées aux États-Unis et $16(29,6 \%)$ étaient des infections contractées en Europe.
} 
Figure 3 : Lieu déclaré d'acquisition de la maladie de Lyme acquise au Canada de 2009 à 2012

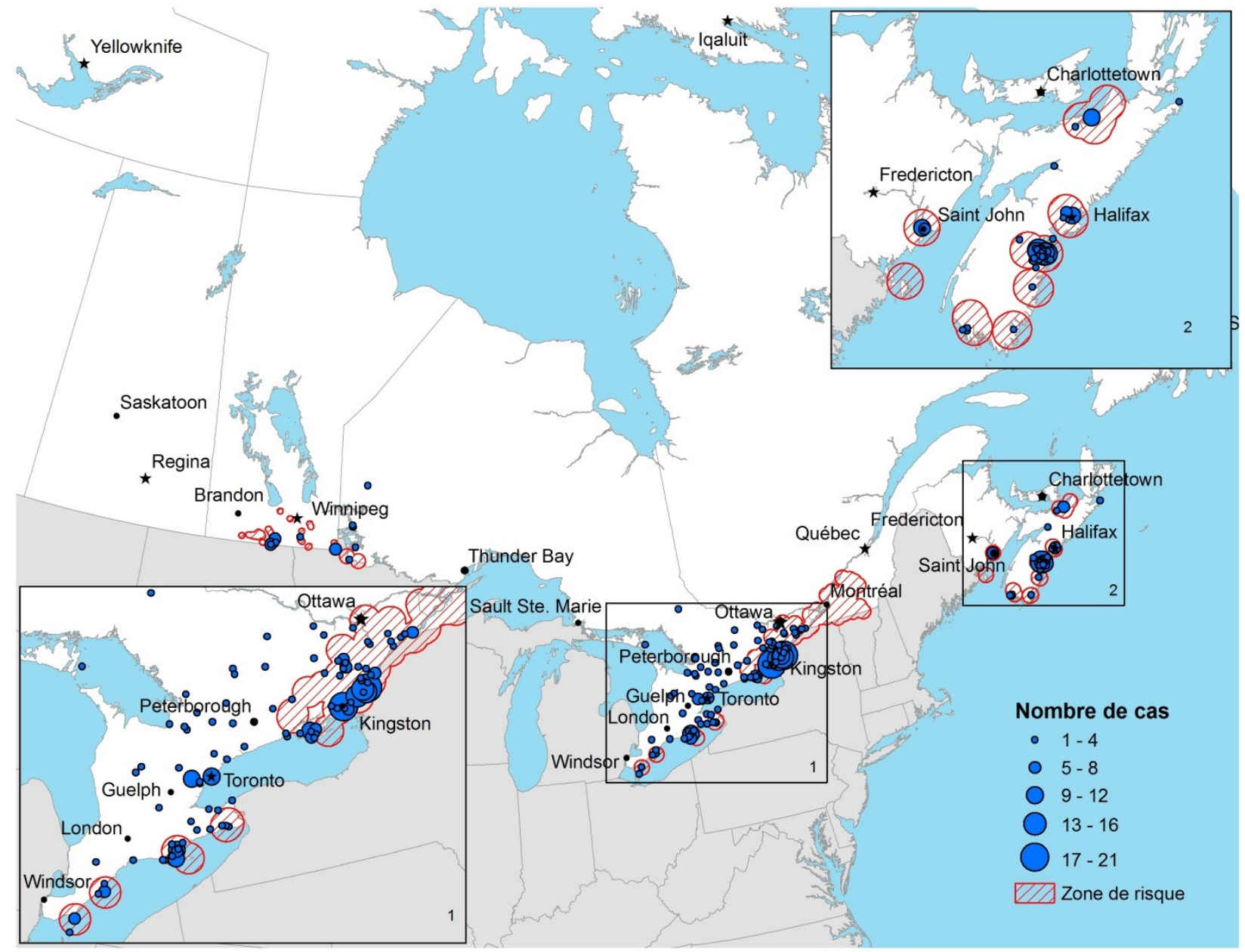

\section{Manifestations cliniques}

Des 353 cas pour lesquels des renseignements sur les cinq catégories de manifestations cliniques étaient disponibles, $157(44,4 \%)$ manifestaient seulement un érythème migrant (donc au stade précoce de la maladie de Lyme) et 92 manifestaient un érythème migrant accompagné de manifestations de la maladie de Lyme au stade disséminé. Les manifestations caractéristiques du stade disséminé précoce de la maladie de Lyme n'ont pas été signalées chez 98 cas $(27,8 \%)$ pour lesquels les symptômes suivants ont été signalés : dans 92 cas $(26,1 \%)$, des manifestations neurologiques ont été signalées (paralysie de Bell signalée chez 30 cas [8,5\%] et d'autres manifestations neurologiques chez 74 cas [21\%]) et des manifestations cardiaques ont été signalées chez 17 cas (4,8 \%) (Figure 4). Les manifestations de l'infection au stade disséminé tardif de la maladie de Lyme, p. ex., l'arthrite, ont été signalées chez 133 cas (37,8\%) (Figure 4). Des manifestations multiples ont été signalées chez 131 cas $(37,2 \%)$. De tous les cas signalés atteints de la forme disséminée de la maladie de Lyme, la proportion des cas associés à des manifestations neurologiques, cardiaques et d'arthrite était respectivement de $38 \%, 7 \%$ et $55 \%$. 
Figure 4 : Pourcentage de cas signalés par la surveillance accrue de la maladie de Lyme associés à différentes manifestations cliniques de la maladie de Lyme comparés à ceux signalés par la surveillance aux États-Unis (10)1

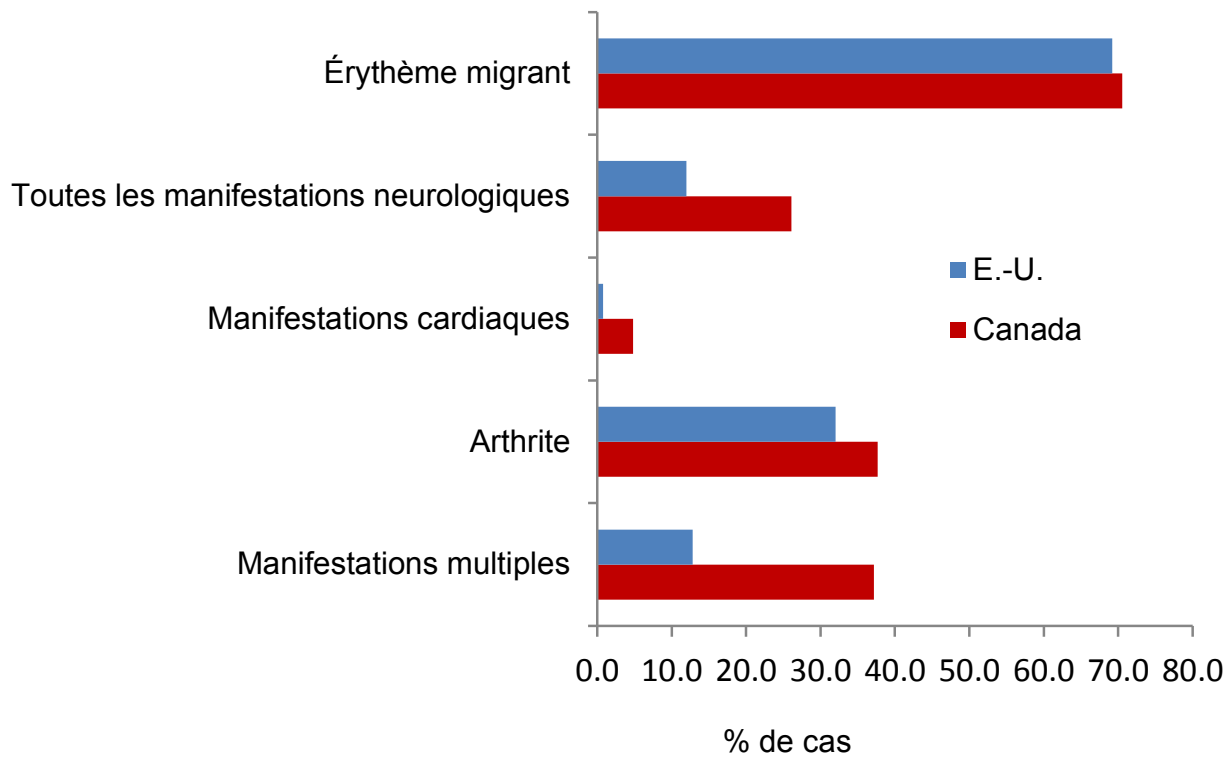

${ }^{1}$ Il est à noter que les valeurs totales pour chaque manifestation sont présentées, et que chaque cas pouvait être associé à des manifestations multiples. La proportion d'ensemble de cas associés à des manifestations multiples est représentée par la plus faible paire de barres.

Selon les définitions de cas aux fins de surveillance, les cas de la maladie de Lyme au stade précoce (la définition de cas « $\mathrm{P} 2$ » : diagnostic d'érythème migrant non confirmé en laboratoire) peuvent seulement être signalés chez des patients ayant été en contact avec des régions d'endémicité de la maladie de Lyme connues. Par conséquent, afin de déterminer la proportion de cas affichant un diagnostic de la maladie de Lyme au stade précoce par opposition au stade disséminé (précoce ou tardif), le dénominateur doit être le nombre de cas pour lesquels l'information sur les manifestations cliniques était disponible et qui ont été contractés dans des zones endémiques connues. II y a eu 302 cas, pour lesquels l'information sur les manifestations cliniques était disponible et qui ont été contractés dans des zones endémiques connues au Manitoba, en Ontario, au Nouveau-Brunswick et en Nouvelle-Écosse. De ces 302 cas, 220 cas $(72,8 \%)$ étaient associés à un érythème migrant, mais l'érythème migrant constituait la seule manifestation chez 120 cas (39,7\%), de sorte que $39,7 \%$ seulement des cas ont été déclarés comme étant au stade précoce de la maladie de Lyme, tandis que le reste $(60,3 \%)$ présentait des symptômes de la maladie de Lyme au stade disséminé (Figure 5). Au Nouveau-Brunswick, les cas d'érythème migrant sans soutien sérologique pour le diagnostic ne sont pas signalés, mais pour quatre des neuf cas $(44,4 \%)$ avec des données sur les manifestations cliniques, un érythème migrant était la seule manifestation clinique. Par conséquent, la possible sous-déclaration de cas probables «P2 » (érythème migrant et contracté dans une région endémique) dans toutes les provinces n'était pas simplement attribuable au manque de signalements au Nouveau-Brunswick. Le nombre de cas au cours de la période de 2009 à 2012 dans les trois provinces les renseignements sur les cinq caractéristiques cliniques (en Ontario, au Nouveau-Brunswick et en Nouvelle-Écosse) a été 275. 
Figure 5 : Pourcentage d'infections contractées dans des régions endémiques signalées à différents stades de la maladie en fonction des manifestations cliniques signalées par la surveillance accrue de la maladie de Lyme

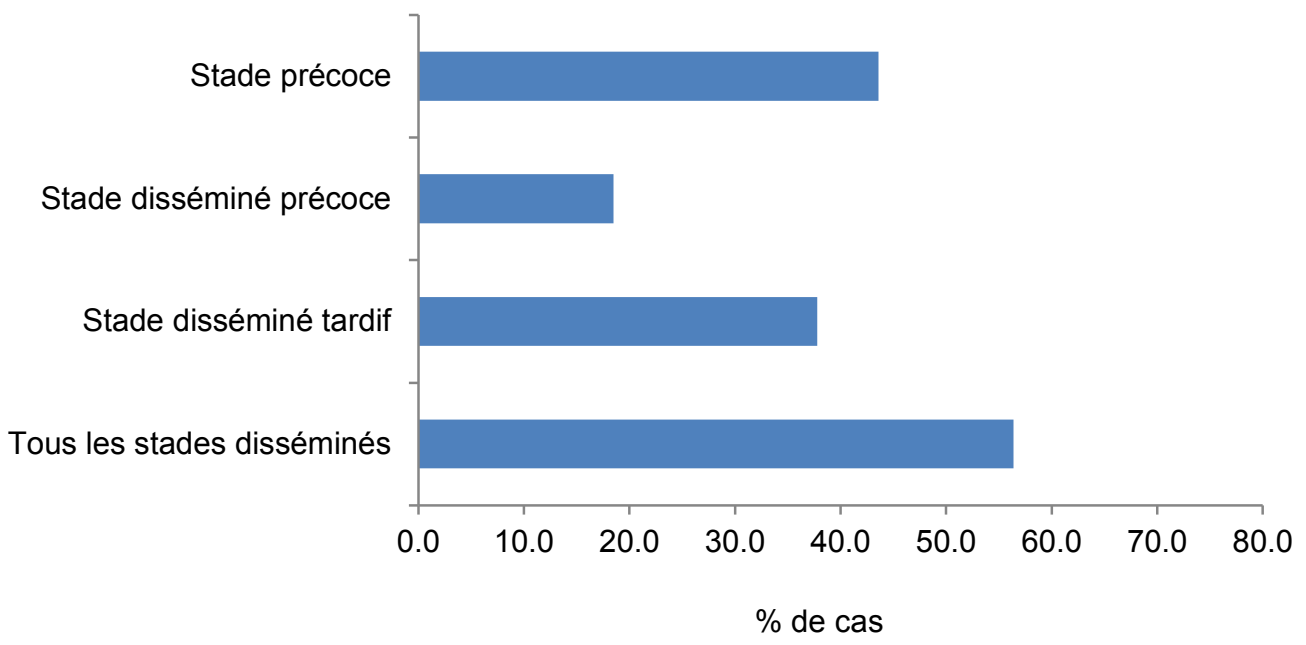

Des cas d'infection contractée dans les régions endémiques, $199(72 \%)$ étaient associés à un érythème migrant (parmi lesquels 120 [43,6 \%] présentaient un érythème migrant comme seule manifestation clinique), 25 (9,1\%) avaient une manifestation de la paralysie de Bell, 60 (21,8 \%) présentaient d'autres symptômes neurologiques (un total de 74 [26,9 \%] ont présenté des symptômes neurologiques de tout genre), 14 (5,1\%) affichaient des symptômes cardiaques et 104 (37,8 \%) présentaient des manifestations d'arthrite ou d'œdème articulaire. Plus d'une manifestation clinique était signalée chez 107 (38,9\%) cas.

Dans l'ensemble, des cas d'infection contractée dans les régions d'endémie, 120 (43,6 \%) étaient au stade précoce de la maladie de Lyme (érythème migrant comme seule manifestation) et 155 cas $(56,4 \%)$ étaient associés à la maladie de Lyme au stade disséminé. Des cas de la maladie de Lyme au stade disséminé, 51 $(18,5 \%)$ étaient du stade disséminé précoce (manifestations neurologiques ou cardiaques, mais pas d'arthrite), et 104 (37,8 \%) étaient du stade disséminé tardif de la maladie de Lyme (avec manifestation d'arthrite).

La fréquence de signalement d'un érythème migrant était la plus élevée chez les enfants et chez les adultes de plus de 50 ans comparativement aux autres groupes d'âge; même après ajustement en fonction de l'année, la fréquence de déclaration d'un érythème migrant augmentait de manière linéaire avec l'âge (Tableau 3, Figure 6). La fréquence des déclarations de manifestations neurologiques et de symptômes cardiaques variait considérablement entre les groupes d'âge. Les manifestations neurologiques étaient les plus fréquemment signalées chez le groupe de 20 à 59 ans et relativement rarement signalées chez les jeunes enfants et chez les adultes âgés de plus de 60 ans, alors que les symptômes cardiaques n'ont été observés que chez les cas âgés de 20 à 69 ans, particulièrement chez les cas âgés de 30 à 49 ans (Figure 6). La proportion de cas signalés affichant des symptômes neurologiques a également été beaucoup plus élevée en Ontario qu'en Nouvelle-Écosse et au Nouveau-Brunswick combinés, et moins élevée chez les femmes que chez les hommes (Tableau 3). II n'y avait pas de différence significative entre les groupes d'âge, les deux sexes, les provinces et les années dans la proportion de cas qui signalaient des manifestations d'arthrite (données non indiquées). 
Tableau 3 : Modèles multivariables finaux, après élimination des variables non significatives $(P>0,05)$, pour lesquelles les variables des résultats étaient la proportion des cas présentant un érythème migrant, des manifestations neurologiques et des manifestations cardiaques.

\begin{tabular}{|c|c|c|c|c|}
\hline Variable & $\begin{array}{l}\text { Rapport de } \\
\text { cotes }\end{array}$ & $\begin{array}{l}\text { Intervalle de confiance à } \\
95 \%\end{array}$ & Wald Z & Valeur P \\
\hline \multicolumn{5}{|c|}{ Résultat = érythème migrant } \\
\hline Année 2010 par rapport à 2009 & 1,364 & $0,616-3,018$ & 0,77 & $>0,1$ \\
\hline Année 2011 par rapport à 2009 & 2,232 & $1,109-4,489$ & 2,25 & $<0,05$ \\
\hline Année 2012 par rapport à 2009 & 2,860 & $1,416-5,778$ & 2,93 & $<0,01$ \\
\hline Âge & 1,015 & $1,004-1,027$ & 2,71 & $<0,01$ \\
\hline \multicolumn{5}{|c|}{ Résultat $=$ manifestations neurologiques } \\
\hline Femmes par rapport aux hommes & 0,561 & $0,345-0,940$ & $-2,19$ & $<0,05$ \\
\hline Âge & 1,073 & $1,014-1,146$ & 2,45 & $<0,05$ \\
\hline Âge carré & 0,998 & $0,998-0,999$ & $-0,30$ & $<0,01$ \\
\hline $\begin{array}{l}\text { Ontario par rapport au Nouveau- } \\
\text { Brunswick et à la Nouvelle- } \\
\text { Écosse. }\end{array}$ & 3,781 & $1,849-7,772$ & 3,62 & $<0,001$ \\
\hline \multicolumn{5}{|c|}{ Résultat = manifestations cardiaques } \\
\hline Âge & 1,305 & $1,043-1,632$ & 2,33 & $<0,05$ \\
\hline Âge carré & 0,997 & $0,994-0,999$ & $-2,41$ & $<0,05$ \\
\hline
\end{tabular}

Figure 6 : Pourcentage de cas, par groupe d'âge présentant un érythème migrant, des manifestations neurologiques, des manifestations cardiaques ou des manifestations d'arthrite ou d'œdème articulaire. ${ }^{1}$

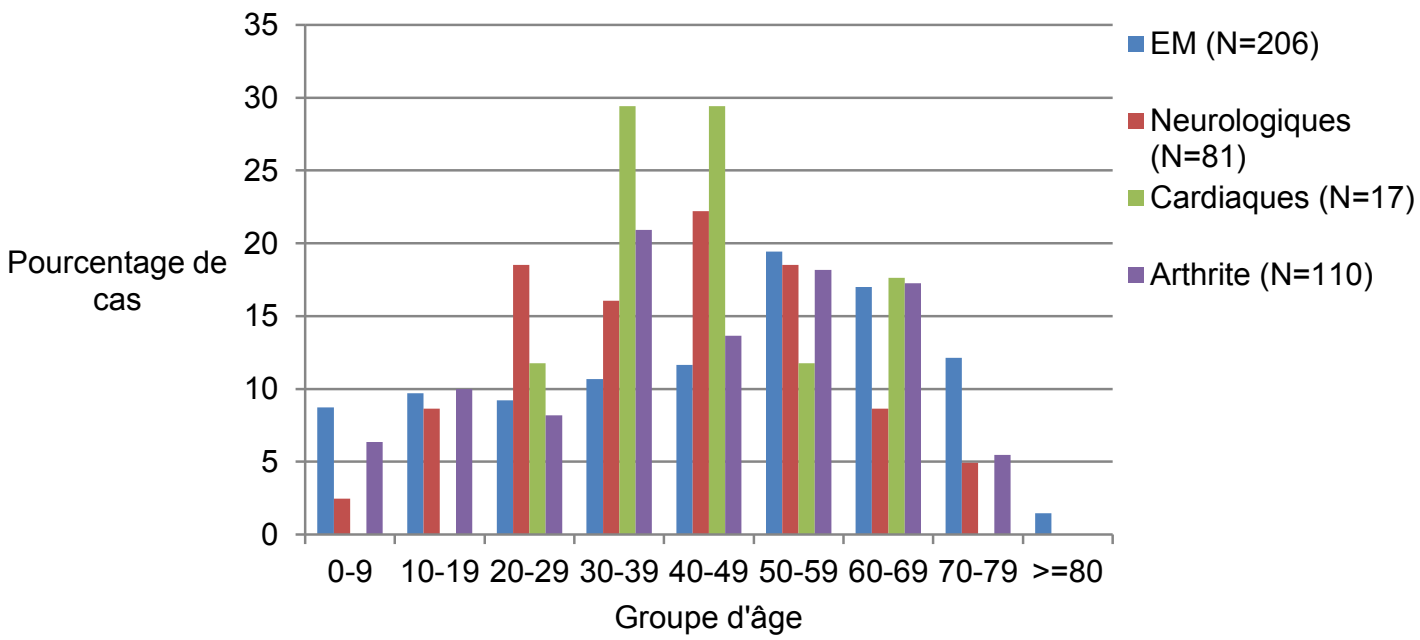

${ }^{1} \mathrm{~N}$ indique le nombre de cas signalés comme ayant des manifestations cliniques de la maladie. 


\section{Discussion}

Le nombre de cas de la maladie de Lyme signalés annuellement au Canada a plus que doublé entre 2009 et 2012. La plus grande part de cette augmentation a été associée à la maladie de Lyme contractée au Manitoba et dans les provinces à l'est du Manitoba. Cette tendance et le profil de variation de l'incidence du VIH entre les provinces est cohérent avec la propagation géographique de l'espèce $I$. scapularis et le risque de maladie de Lyme dans le centre et l'est du Canada, malgré qu'une plus grande sensibilisation du public et des professionnels de la santé puisse entraîner une plus grande proportion de cas signalés. Le nombre de cas signalés de la maladie de Lyme constitue probablement une sous-estimation de la réalité, en raison de la probabilité de sous-déclaration des cas de la maladie de Lyme dans les zones émergentes (13) et en raison du fait que les cas au stade précoce de la maladie de Lyme) ne peuvent être signalés que s'ils ont été acquis dans les régions endémiques, à moins d'être confirmés en laboratoire. Les cas d'infections contractées à l'extérieur de ces zones ne sont pas déclarés aux autorités de santé publique.

Les deux tiers des cas de maladie de Lyme ont été " confirmés », ce qui est cohérent avec le fait que le risque de contracter la maladie de Lyme est particulièrement élevé dans les régions d'endémicité. La surveillance des tiques $(3,14,15)$ indique que la population d'Ixodes scapularis élargit sa portée géographique au Canada et près d'un quart des cas de la maladie de Lyme au stade disséminé ont été contractés dans des zones non connues comme étant endémiques (et étaient des cas probables P1). Toutefois, la plupart de ces cas sont survenus dans des zones où les populations d'l. scapularis sont émergentes (Figure 3). Dans les régions où la maladie est endémique, les risques et l'incidence pourraient être beaucoup plus élevés (>25/100 000) que les valeurs provinciales d'incidence (16). Un petit nombre de cas de la maladie de Lyme ont été observés dans des régions où des populations d'l. scapularis n'ont pas encore été recensées, mais où peu de surveillance sur le terrain a eu lieu à ce jour afin d'en vérifier la présence ou l'absence.

L'incidence des cas déclarés chez les adultes était la plus élevée chez les personnes âgées de plus de 54 ans, chez les hommes et chez les enfants de moins de 15 ans. Cette tendance correspond à la surveillance aux États-Unis (10). Ces groupes d'âge, et les hommes, peuvent être particulièrement à risque de contracter la maladie de Lyme. Cependant, les enfants et les personnes âgées ont fait l'objet d'un diagnostic de la maladie de Lyme au stade précoce plus fréquemment, et moins fréquemment au stade disséminé, de sorte qu'une incidence élevée chez ces groupes peut être le reflet d'une plus grande sensibilisation et du fait de se présenter plus tôt pour obtenir un diagnostic en comparaison des aux jeunes adultes.

Le caractère saisonnier des cas au Canada était semblable à ce qui a été observé aux États-Unis (10) et était conforme aux données sur la transmission transmise par les tiques. $I$. scapularis et $I$. pacificus sont actives d'avril à novembre, et les activités récréatives de plein air dans des régions boisées sont aussi plus susceptibles d'avoir lieu pendant cette période. Les tiques au stade nymphal transmettent la plupart des infections à la maladie de Lyme (17), mais il y a eu des cas où l'infection a été contractée au début du printemps et à l'automne lorsque les tiques adultes sont les plus actives. Compte tenu de la plus longue saison d'activité des tiques I. pacificus comparativement à $I$. scapularis (18), un plus grand nombre de cas a été signalé plus tôt et plus tard dans l'année en Colombie-Britannique où I pacificus est responsable de la transmission vectorielle. Les délais entre l'infection et le diagnostic de la maladie de Lyme au stade disséminé pourraient expliquer le signalement de quelques cas au cours de l'hiver (9), bien que la date d'apparition déclarée de certains cas de la maladie de Lyme au stade précoce se situe au cours de l'hiver. Les raisons pour lesquelles plusieurs cas de la maladie de Lyme ont été signalés à l'automne au Manitoba, comparativement aux autres provinces, ne sont pas claires, puisque les tiques devraient également être actives dans les autres provinces à ce moment.

Les données sur les types de maladie de Lyme déclarés et provenant de zones endémiques suggèrent qu'il existe un phénomène de sensibilisation sous-optimale à l'égard de la maladie de Lyme parmi la population et les professionnels de la santé de première ligne. Des antécédents d'érythème migrant ont été signalés chez plus de $80 \%$ des cas d'infection contractée dans les zones endémiques connues. Toutefois, seulement $40 \%$ des cas ont été signalés au cours du stade précoce de la maladie de Lyme, et $60 \%$ étaient des cas probables de la maladie de Lyme au stade disséminé, et ce, même si un érythème migrant avait été déclaré comme manifestation dans 
de nombreux cas de la maladie de Lyme au stade disséminé. Cela laisse à penser que bon nombre de cas de la maladie de Lyme au stade disséminé auraient pu être diagnostiqués et traités plus tôt, mais soit les patients touchés ne savaient pas ce qu'était l'érythème migrant (et n'ont pas tenté d'obtenir un diagnostic à ce stade), soit les professionnels de la santé n'ont pas diagnostiqué et traité les cas à ce stade. Aux États-Unis, où la sensibilisation du public et des professionnels de la santé devrait être plus importante, plus de $56 \%$ des cas signalés étaient des cas au stade précoce de la maladie de Lyme (10).

La proportion globale des manifestations cliniques de la maladie de Lyme au stade disséminé signalé au Canada était semblable à celle des États-Unis. Lorsque les proportions de cas au stade disséminé montrant des manifestations neurologiques, cardiaques et d'arthrite (c.-à-d. le stade tardif de la maladie de Lyme) ont été comparées à des données similaires provenant des États-Unis (10), il y avait certaines différences. Les proportions de cas au stade disséminé tardif de la maladie étaient semblables (55\% et $50 \%$ pour le Canada et les États-Unis, respectivement), mais la proportion de cas affichant des symptômes neurologiques était plus faible au Canada (38\% par rapport à $47 \%$ aux États-Unis) et la proportion affichant des manifestations cardiaques était plus élevée au Canada (7\% par rapport à $3 \%$ aux États-Unis). Plus de données de surveillance sont nécessaires pour déterminer s'il s'agit d'une différence uniforme et s'il y a des changements au fil du temps. Effectuer un suivi de la fréquence de la cardite de Lyme est également important, car elle a été associée à des décès soudains (19).

Les manifestations neurologiques et cardiaques étaient plus susceptibles d'être signalées chez les jeunes adultes, alors qu'il n'y avait pas de preuve de corrélation entre l'âge et les variations dans la fréquence de signalement des manifestations d'arthrite. Les manifestations neurologiques étaient moins susceptibles d'être signalées chez les hommes que chez les femmes. Les raisons expliquant ces observations ne sont pas claires et nécessitent des recherches plus approfondies. Le signalement des symptômes neurologiques était plus courant en Ontario que dans la région des Maritimes probablement en raison de méthodes différentes de déclaration des symptômes entre les provinces, ou en raison d'une variation géographique dans les souches de

B. burgdorferi (20).

Les observations et les résultats de cette étude représentent un premier aperçu des données de surveillance de la maladie de Lyme au Canada, et il est trop tôt pour tirer des conclusions irréfutables de ces tendances préliminaires. Il est possible que les données canadiennes sur le lieu de l'acquisition et sur la manifestation de l'infection soient influencées par les problèmes de rappel et autres inexactitudes. Ces constatations requièrent une étude plus poussée pour être corroborées et pour évaluer la causalité.

\section{Conclusion}

Ces données laissent entendre que la maladie de Lyme est en émergence au Canada, la plupart des cas survenant au cours des saisons et dans les régions où le risque d'occurrence de la maladie de Lyme dans l'environnement est connu. L'incidence était plus élevée chez les hommes, chez les adultes de plus de 54 ans et chez les enfants de moins de 15 ans. La proportion de cas signalés au stade précoce de la maladie de Lyme était plus faible que prévu, ce qui laisse supposer une sensibilisation sous-optimale à l'égard de la maladie de Lyme au cours de la période de surveillance. Des variations entre les provinces et les groupes d'âge concernant la proportion de cas associés à l'apparition d'un érythème migrant et à des manifestations neurologiques et cardiaques du stade disséminé de la maladie de Lyme ont été relevées, mais demeurent à l'heure actuelle inexpliquées. 


\section{Remerciements}

Les auteurs remercient Yann Pelcat de l'Agence de la santé publique du Canada pour la préparation de la Figure 3.

\section{Conflit d'intérêts}

Aucun

\section{Références}

(1) Ogden NH, Lindsay RL, Sockett PN, Morshed M, Artsob H. Emergence of Lyme disease in Canada. CMAJ. 2009;180:1221-4.

(2) Kurtenbach K, Hanincová K, Tsao J, Margos G, Fish D, Ogden NH. Key processes in the evolutionary ecology of Lyme borreliosis. Nat Rev Microbiol. 2006;4:660-9.

(3) Ogden NH, Koffi, JK, Pelcat Y, Lindsay LR. Environmental risk from Lyme disease in central and eastern Canada: A summary of recent surveillance information. Can Commun Dis Rep. 2014;40:74-82.

Disponible en français: http://www.phac-aspc.gc.ca/publicat/ccdr-rmtc/14vol40/dr-rm40-05/dr-rm40-05-1-fra.php.

(4) Ogden NH, Lindsay RL, Hanincová K, Barker IK, Bigras-Poulin M, Charron DF, et al. The role of migratory birds in introduction and range expansion of Ixodes scapularis ticks and Borrelia burgdorferi and Anaplasma phagocytophilum in Canada. Appl Environ Microbiol. 2008;74:1780-90.

(5) Vrbova L, Middleton D. Descriptive epidemiology of Lyme disease in Ontario: 1999-2004. Can Commun Dis Rep. 2006;32:247-57.

(6) Public Health Agency of Canada. Case definitions for communicable diseases under national surveillance. Can Commun Dis Rep. 2009;35:S2.

Disponible en français: http://www.phac-aspc.gc.ca/publicat/ccdr-rmtc/09vol35/35s2/index-fra.php.

(7) Canadian Public Health Laboratory Network. The laboratory diagnosis of Lyme borreliosis: Guidelines from the Canadian Public Health Laboratory Network. Can J Infect Dis Med Microbiol. 2007;18:145-8.

(8) Hatchette TF, Davis I, Johnston BL. Lyme disease: Clinical diagnosis and treatment. Can Commun Dis Rep. 2014;40-11:194-208.

Disponible en français: http://www.phac-aspc.gc.ca/publicat/ccdr-rmtc/14vol40/dr-rm40-11/dr-rm40-11-lyme-1fra.php.

(9) Wormser GP, Dattwyler RJ, Shapiro ED, Halperin JJ, Steere AC, Klempner MS, et al. The clinical assessment, treatment, and prevention of Lyme disease, human granulocytic anaplasmosis, and babesiosis: Clinical practice guidelines by the Infectious Diseases Society of America. Clin Infect Dis. 2006;43:1089-134.

(10) Bacon RM, Kugeler KJ, Mead P. Surveillance for Lyme disease United States, 1992-2006. MMWR Surveill Sum. 2008;57:1-9.

(11) Statistics Canada. CANSIM census data. 2014. http://www5.statcan.gc.ca/cansim/a33?lang=eng\&spMode=master\&themelD=3867\&RT=TABLE. Disponible en français:

http://www5.statcan.gc.ca/cansim/a33?themeID=3867\&RT=TABLE\&spMode=master\&retrLang=fra\&lang=fra

(12) Statistics Canada [Internet]. Population by year, by province and territory. 2014. http://www.statcan.gc.ca/tablestableaux/sum-som/101/cst01/demo02a-eng.htm.

Disponible en français: http://www.statcan.gc.ca/tables-tableaux/sum-som/l02/cst01/demo02a-fra.htm.

(13) Naleway AL, Belongia EA, Kazmierczak JJ, Greenlee RT, Davis JP. Lyme disease incidence in Wisconsin: A comparison of state-reported rates and rates from a population-based cohort. Am J Epidemiol. 2002;155:1120-7.

(14) Leighton P, Koffi J, Pelcat Y, Lindsay LR, Ogden NH. Predicting the speed of tick invasion: An empirical model of range expansion for the Lyme disease vector Ixodes scapularis in Canada. J Appl Ecol. 2012;49:457-64.

(15) Ogden NH, Lindsay LR, Leighton P. Predicting the rate of invasion of the agent of Lyme disease, Borrelia burgdorferi in North America. J Appl Ecol. 2013;50:510-8.

(16) Public Health Ontario. 2013 Vector-borne Diseases: 2013 Summary Report. Toronto, ON: Queen's Printer for Ontario; 2012.

http://www.publichealthontario.ca/en/eRepository/Vector_Borne_Diseases_Summary_Report_2013.pdf.

(17) Falco RC, McKenna DF, Daniels TJ, Nadelman RB, Nowakowsk̄i J, Fish D, et al. Temporal relation between Ixodes scapularis abundance and risk for Lyme disease associated with erythema migrans. Am J Epidemiol. 1999;149:7716.

(18) Salkeld DJ, Castro MB, Bonilla D, Kjemtrup A, Kramer VL, Lane RS, et al. Seasonal activity patterns of the western black-legged tick, Ixodes pacificus, in relation to onset of human Lyme disease in northwestern California. Ticks Tick Borne Dis. 2014;5:790-6. 
(19) Centers for Disease Control and Prevention. Three sudden cardiac deaths associated with Lyme carditis - United States, November 2012-July 2013. MMWR Morb Mortal Wkly Rep. 2013;62:993-6.

(20) Mechai S, Margos G, Feil EJ, Lindsay LR, Ogden NH. Phylogeographic analysis reveals a complex population structure of Borrelia burgdorferi in southern Canada. Appl Env Microbiol. 2015; In press. 
Annexe 1 : Données recueillies dans le cadre de la surveillance nationale de la maladie de Lyme au Canada au cours de la période de 2009 à 2012

\begin{tabular}{|c|c|c|}
\hline Description des données & Type de données & Provinces ayant fourni des données \\
\hline Âge & Continues & Tous \\
\hline Sexe & Hommes/Femmes & Tous \\
\hline Classification des cas & Confirmés/Probables & $\begin{array}{c}\text { Alb., Sask., Man., Ont., Î.-P.-É., N.-É. } \\
\text { et T.-N.-L. }\end{array}$ \\
\hline Date de l'épisode & Jour, Mois, Année & Tous \\
\hline Date du type d'épisode ${ }^{1}$ & $\begin{array}{l}\text { Catégorie : Apparition/Collecte } \\
\text { d'échantillon/Diagnostic/Rapport }\end{array}$ & Man., Ont., N.-B., N.-É. et Î.-P.-É ${ }^{5}$ \\
\hline Voyages à l'extérieur du Canada & Oui/Non & $\begin{array}{l}\text { Alb., Sask., Man., Ont., N.-B., Î.-P.-É., } \\
\text { N.-É., T.-N.-L. }\end{array}$ \\
\hline $\begin{array}{l}\text { Exposition à une région endémique } \\
\text { connue au Canada au cours des } \\
30 \text { derniers jours }{ }^{1}\end{array}$ & Oui/Non & Man., Ont., N.-B., N.-É. \\
\hline $\begin{array}{l}\text { Nom/identifiant de la région endémique au } \\
\text { Canada }^{1}\end{array}$ & Géolocalisateur & \\
\hline $\begin{array}{l}\text { Exposition à une région endémique } \\
\text { connue au Canada au cours des } \\
30 \text { derniers jours }{ }^{1}\end{array}$ & Oui/Non & Man., Ont., N.-B., N.-É. \\
\hline $\begin{array}{l}\text { Nom/identifiant de la région endémique à } \\
\text { l'extérieur du Canada }{ }^{1}\end{array}$ & Géolocalisateur & Man., Ont., N.-B., N.-É. \\
\hline $\begin{array}{l}\text { Région de résidence de tri } \\
\text { d'acheminement (RTA : trois premiers } \\
\text { caractères du code postal) }{ }^{1,2}\end{array}$ & Géolocalisateur & Man., Ont., N.-B., N.-É. \\
\hline $\begin{array}{l}\text { Symptômes de la maladie de Lyme au } \\
\text { stade précoce (érythème migrant) }\end{array}$ & Oui/Non & Man., Ont., N.-B., N.-É. \\
\hline $\begin{array}{l}\text { Symptômes de la maladie de Lyme au } \\
\text { stade disséminé }^{1}\end{array}$ & Oui/Non & Man., Ont., N.-B., N.-É. \\
\hline $\begin{array}{l}\text { Symptômes de la maladie de Lyme au } \\
\text { stade disséminé : paralysie de Bell }\end{array}$ & Oui/Non & Ont., N.-B., N.-É. ${ }^{\top}$ \\
\hline $\begin{array}{l}\text { Symptômes de la maladie de Lyme au } \\
\text { stade disséminé : autres symptômes } \\
\text { neurologiques }\end{array}$ & Oui/Non & Ont., N.-B., N.-É. ${ }^{1}$ \\
\hline $\begin{array}{l}\text { Symptômes de la maladie de Lyme au } \\
\text { stade disséminé : troubles cardiaques }{ }^{1,4}\end{array}$ & Oui/Non & Ont., N.-B., N.-É.' \\
\hline $\begin{array}{l}\text { Symptômes de la maladie de Lyme au } \\
\text { stade tardif : œdème articulaire ou arthrite } \\
\text { récurrents }\end{array}$ & Oui/Non & Ont., N.-B., N.-É. \\
\hline Méthode de diagnostic $^{1}$ & $\begin{array}{l}\text { Catégorie : Sérologie/Test de } \\
\text { polymérase en chaîne/Culture }\end{array}$ & Man., Ont., N.-B., N.-É.* \\
\hline
\end{tabular}

${ }^{1}$ Les données recueillies dans le cadre du système de surveillance accrue de la maladie de Lyme sont indiquées par un astérisque. Sinon, les données ont été recueillies par l'entremise du Système canadien de surveillance des maladies à déclaration obligatoire.

${ }^{2}$ La région de tri d'acheminement de résidence a été considérée comme le lieu de l'acquisition en l'absence d'antécédents d'exposition ou de voyage enregistrés dans une zone à risque connue de la maladie de Lyme au Canada ou à l'étranger.

${ }^{3}$ Radiculoneuropathie, encéphalite, méningite lymphoblastique et encéphalomyélite.

${ }^{4}$ Blocage cardiaque atrioventriculaire et myocardite.

${ }^{5}$ L'Île-du-Prince-Édouard a fourni des éléments de données de surveillance accrue de la maladie de Lyme pour 2012 en août 2014; par conséquent, ces renseignements n'avaient pas été pris en compte lors de l'analyse. 